\title{
Hierarchical-Battery Aware Routing in Wireless Sensor Networks
}

\author{
Ravi Musunuri Jorge A. Cobb \\ Department of Computer Science \\ The University of Texas at Dallas \\ Richardson, TX-75083-0688 \\ Email: \{musunuri,cobb\}@utdallas.edu
}

\begin{abstract}
The Wireless Sensor Networks (WSN) have been envisioned to help in numerous monitoring applications. Pulsed battery discharge from the nodes prolongs the battery capacity as compared to constant discharge. Idle time between the pulses of discharge helps in recovering the discharged battery capacity. In the hierarchical routing protocol, cluster head nodes expend much higher energy than other nodes without any idle time.

In this paper, we are proposing a Hierarchical-Battery Aware Routing (H-BAR) protocol for the WSNs. To make discharge from the nodes as pulsed as possible, role of cluster heads is changed periodically between the nodes. Battery recovery capacity depends on the remaining capacities of the battery. Nodes with the higher remaining capacities will have a higher probability of recovering than the nodes with the lower remaining capacities. Hence, to improve the recovery capacity, discharge from each node should be as uniform as possible. In the previous hierarchical routing protocols, such as LEACH, periodic selection of cluster heads is done probabilistically. Probabilistic election does not provide uniform discharge from the nodes. In the H-BAR, to provide uniform discharge, nodes with the higher remaining capacities are chosen as the cluster heads. Simulations show that H-BAR can improve the lifetime of the WSN up to 3 times over the lifetime of the WSN using the LEACH protocol.
\end{abstract}

\section{INTRODUCTION}

The Wireless Sensor Networks (WSN) have been envisioned to help in numerous monitoring applications [1], [2] in both military and civilian fields. The WSN consists of thousands of small and cheap nodes, when deployed, should organize themselves, detect specific event(s) and send this information to the sink node. Each sensor node consists of a small battery, a sensing device, a radio device with an amplifier, and other digital circuitry. In a typical sensor node, sensing device and digital circuitry draw a very small amount of constant power from the battery. But the radio device with an amplifier draws much higher power during the transmission and the reception of the packets. Hence, designing a energy efficient routing protocol is paramount to improve the lifetime of the WSN.

Every node is powered with a limited capacity battery. Battery discharge characteristics also greatly influence the capacity of the battery. There are two types of battery capacities: theoretical capacity and nominal capacity, which are explained in the Section II. Several works [3], [4] have showed that the battery capacity can be prolonged up to the theoretical capacity [5] by using pulsed discharge instead of constant discharge. Idle time between the pulses of discharge allows the battery to recover some of its spent nominal capacity

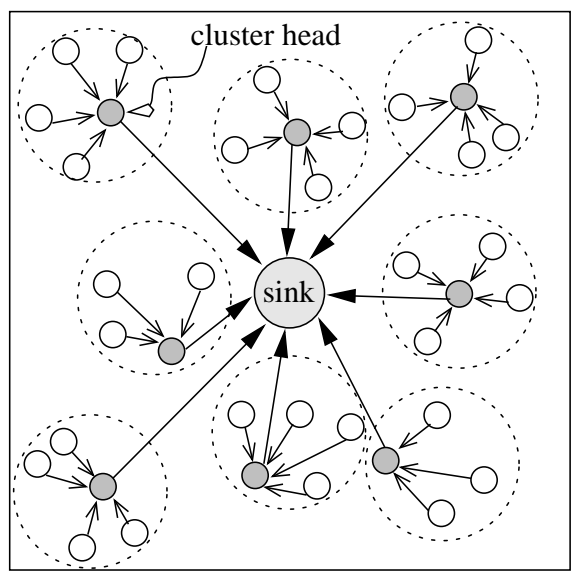

Fig. 1. Example Sensor Field with Clusters

due to recovery capacity effect [5]. Recovery capacity of the battery depends on the state of the battery. The state of the battery [5] is represented as a 2-tuple, $\left\langle T_{i}, N_{i}\right\rangle$, where $T_{i}$, and $N_{i}$ denote the remaining theoretical and nominal battery capacities respectively.

Current WSN routing protocols can be grouped into following two categories: hierarchical [6], [7], [8] and flat [9], [10]. In the hierarchical routing, nodes are divided into logical clusters. In each cluster, some special node acts as the cluster head. Other nodes inside the cluster are referred as the clients. Data packets originating from the client node are forwarded to its cluster head. Based on the type of application, cluster head aggregates the data packets before forwarding them to the sink node. In the hierarchical routing, cluster head needs to expend much higher energy than the client nodes without any idle times. Flat routing does not have any special cluster head nodes. Every node should forward its data packets towards the sink node.

In this paper, we are proposing a Hierarchical-Battery Aware Routing (H-BAR) protocol for the WSNs. H-BAR is a distributed protocol, in which, role of cluster heads is changed between nodes periodically. Due to periodic changing, every node expends its energy in a pulsed pattern. To make discharge as uniform as possible, nodes with the higher remaining capacities are chosen as the cluster heads. Previous hierarchical routing protocols [6], [7], [8] does not provide 
uniform discharge from the nodes. We are not aware of any work that incorporates the battery awareness in the hierarchical routing. We will show through extensive simulations that the H-BAR improves the lifetime of the network up to 3 times over the LEACH protocol.

\section{BATTERY CHARACTERISTICS}

There are two types of battery capacities, which are defined as below:

- Theoretical Capacity (T) : The battery capacity available from the total active materials contained in the battery. Hence, the total battery discharge can't be more than the theoretical capacity.

- Nominal (Standard) Capacity (N): This corresponds to the actual capacity available, when the battery is discharged at a specified constant current.

Capacity of the battery depends on the following two important characteristics.

\section{A. Rate Capacity Effect}

The rate, at which, the battery discharges has profound impact on the nominal capacity of the battery. Batteries discharged at lower rate will be able to deliver higher capacity than the batteries discharged at higher rate. Many papers have showed that the pulsed discharge [3], [4], [11] from the battery increases the nominal battery capacity over the constant discharge.

\section{B. Recovery capacity Effect}

In the pulsed discharge, if the battery gets enough idle time between pulses of discharge, then the battery can recover some of its discharged nominal capacity. Due to recovery effect, we can completely use the theoretical capacity of the battery by smartly controlling the battery discharge characteristics. Recovery effect depends on the remaining nominal and theoretical capacities of the battery.

\section{SySTEM MODEL}

We assume the following system model, which is similar to the system model used in [6], [7], [8].

- Sensor nodes are static and placed randomly over a square area sensing field.

- Every sensor node senses an event and sends this information back to a static sink node, which is located at the center of the sensing field.

- Every node is equipped with a smart battery [12], from which, each node can read the remaining theoretical and nominal capacities.

- Every sensor node is time synchronized [13], [14].

- WSN lifetime is divided into pre-defined, equal size periods as shown in the Fig. 2, which is similar to the round used in [6], [7].

- Similar to the LEACH [6], [7], our protocol assumes CDMA-TDMA communication scheme, i.e., each cluster uses different CDMA code and the communication between cluster head and its client nodes uses TDMA slots to avoid contention.

Next, we will explain the radio propagation, and the battery recovery model assumed in this paper.

\section{A. Radio Propagation Model}

Energy required to make a successful data transmission between neighboring nodes depends on the radio propagation model. In this paper, we are assuming the first order radio model [6], [7], [8]. In this model, energy required to transmit the data is sum of the energy required to run the transmitter circuitry and the energy required to run the amplifier circuitry. Energy required to receive the data is equal to the energy required to run the receiver circuitry. Both the transmitter and the receiver spend constant energy (50 nano Jules/bit). Amplifier circuitry spends 100 pico Jules/bit energy to each $m^{2}$ area broadcast.

\section{B. Battery Recovery Model}

We assume that every node recovers 0.05 micro Jules of nominal capacity during the idle recovery period with recovery probability $\left(R P_{i, j}\right)$. Recovery period depends on the type of the battery. Recovery probability [5] is a decreasing exponential function as given below:

$$
R P_{i, j}= \begin{cases}e^{-\left(g *\left(N-N_{i}\right)+\phi\left(T_{i}\right)\right)} & \text { if } 0 \leq N_{i} \leq N \\ 0 & \text { and } 0 \leq T_{i} \leq T \\ & \text { otherwise }\end{cases}
$$

Where, $N$ and $T$ are the maximum nominal and theoretical capacities respectively. Battery parameter $g$ is a constant and $\phi\left(T_{i}\right)$ is a piece-wise constant function [5] as given below.

$$
\phi\left(T_{i}\right)= \begin{cases}15.6 & \text { if } 0 \leq T_{i}<0.025 * T \\ 0.08 & \text { if } 0.025 * T \leq T_{i}<0.5 * T \\ 0.025 & \text { if } 0.5 * T \leq T_{i}<0.975 * T \\ 0 & \text { if } 0.975 * T \leq T_{i}<T\end{cases}
$$

\section{RELATED WORK}

In each round, LEACH protocol [6], [7] selects the clusters heads probabilistically. Each node, $i$, elects itself as the cluster head by selecting a random number between zero to one and calculating following threshold value, $T(i)$.

$$
T(i)= \begin{cases}\frac{P}{1-P *\left(r \bmod \frac{1}{P}\right)} & \text { if node } i \in G \\ 0 & \text { otherwise }\end{cases}
$$

Where $P$ is the desired percentage of cluster heads in each round and the set $G$ stores the ids of the nodes that became the cluster heads during the last $\frac{1}{P}$ rounds. If the generated random number is less than $T(i)$ then the node, $i$, elects itself as the cluster head.

LEACH protocol improves the lifetime [6], [7] of the WSN, when compared with the direct transmission and the transmission along the minimum energy spanning tree. In the direct transmission, every node directly transmits its data packets to the sink node. But, the LEACH protocol has the following 


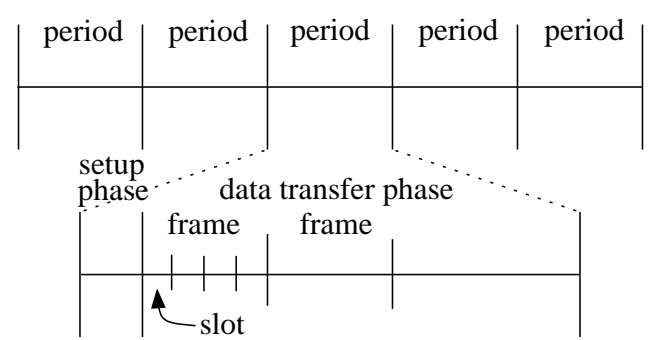

Fig. 2. Division of WSN Lifetime

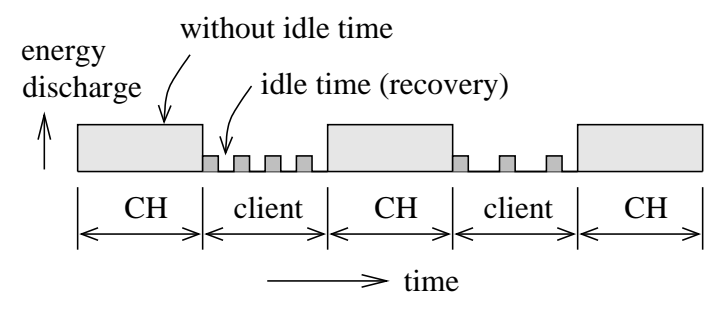

Fig. 3. Illustration of Pulsed Discharge from a Node disadvantage. As discussed in the next section V, LEACH protocol does not provide uniform discharge from the sensor nodes. In [8], authors proposed a Stable Election Protocol (SEP) for the heterogeneous WSNs. In the heterogeneous WSNs, initial battery capacities at all sensor nodes are not the same. SEP is similar to LEACH, but each node $i$, while calculating the threshold $T(i)$, also considers the heterogeneity in the battery capacities. Similar to LEACH, SEP also may not provide uniform discharge from the sensor nodes.

Battery discharge characteristics also greatly influence the lifetime of the WSNs. In [15], authors proposed a Battery Aware Medium Access Protocol (BAMAC) to improve the lifetime in ad-hoc wireless networks. BAMAC improves the lifetime by smartly controlling the discharge from each node. Chiasserini and Rao [12], [16], [17] have studied many problems on using the pulsed battery discharge to improve the lifetime of the battery.

\section{HierarchicAl-BATtery AWARE Routing (H-BAR)}

The H-BAR is a energy efficient hierarchical routing protocol for the WSNs. To prolong the lifetime of the WSN, routing protocol should have the following two important properties. Firstly, routing protocol should drain the battery at each node in the pulsed fashion. As discussed before, pulsed battery discharge prolongs the lifetime of the battery as compared to the constant discharge due to recovery capacity effect. Idle time, between the pulses of discharge, helps the battery to recover some of its used nominal capacity. From the equation 1 , we can see that the recovery probability is a exponentially decreasing function of the remaining nominal and theoretical capacities. Recovery probability is higher, when the node has the higher remaining capacities. Recovery probability decreases exponentially as the remaining capacities decrease. Secondly and most importantly, energy discharge from the battery of each sensor node should be as uniform as possible. Uniform discharge increases the recovered battery capacity in the WSN.

To make pulsed discharge from each sensor node, WSN lifetime is divided into pre-defined, equal size periods as shown in the Fig. 2. In the beginning of every period, new set of cluster heads are selected. We know that the cluster head nodes expend much higher energy without any idle time. But, the client nodes expend much lower energy with idle time. Length of the idle time depends on the TDMA schedule in each cluster. Illustration of the energy drain from the battery at a node is presented in the Fig. 3. Hence, the periodic changing of cluster head role between different nodes in each period helps to drain the battery in the pulsed pattern.

Periodic changing of cluster heads has been used in the previous routing protocols [6], [7], [8] to spread the battery discharge to all the nodes in the WSN. But the previous routing protocols might not provide the uniform discharge from each node due to following reason. During the lifetime of the WSN, each node has an equal probability of becoming a cluster head. Each node, when it takes up the cluster head role, spends a different amount of energy based on its distance from the sink node. Similarly, each node, when it is a client node, spends a different amount of energy based on its distance from its cluster head. To make the battery discharge as uniform as possible, nodes with the higher remaining capacities should have a higher probability of becoming a cluster head in each period than the nodes with the lower remaining capacities.

Each period begins with a shorter duration cluster setup phase followed by a longer duration data transfer phase. Data transfer phase duration has also been divided into pre-defined, equal size slots as shown in the Fig. 2.

During the cluster setup phase, new set of cluster heads gets elected based on the remaining capacities at each node. Average number of cluster heads per period also plays an important role on the lifetime of the WSN. Every node in the WSN acting as the cluster head is same as zero nodes acting as the cluster head. In both the cases [6], every node sends its data packets directly (direct transmission) to the sink node and the lifetime of the WSN is the lowest. As the average number of cluster heads per period varied from zero to the maximum number (i.e., all the nodes in the WSN), the lifetime of the WSN [6] reaches a maximum value before reaching the lowest value again. As noticed in [6], [8], we also noticed an exponential decrease in the lifetime of the WSN as the average number of cluster heads per period increased after reaching the maximum lifetime value.

To control the average number of cluster heads per period, $\mathrm{H}-\mathrm{BAR}$ has a transmission range value, which is denoted as $D$. This range value is a input parameter to the H-BAR protocol. Higher the $D$ value, lower the average cluster size per period and vice versa. Optimal cluster size value can be found from the theoretical analysis [18], which can be used as the basis for deciding the input parameter $D$.

Cluster setup phase consists of the following four steps. Steps three and four are similar to the LEACH protocol. 
1) Battery State Announcement (BSA): Every node broadcasts its remaining theoretical $\left(T_{i}\right)$, and nominal capacities $\left(N_{i}\right)$ within the $D$ range.

2) Cluster Head Announcement (CHA): Every node elects itself as the cluster head, if it has the highest theoretical capacity among the nodes from which it received the BSA message. Each elected cluster head node advertises its intention of becoming a cluster head within the $D$ range. Ties in the theoretical capacities are broken by using the nominal capacities, and the node ids. LEACH protocol requires each sensor node to advertise its CHA message with the maximum range, which might be much higher than the $D$ value. Hence, this step in the LEACH protocol discharges much higher energy than our H-BAR protocol. We did not considered the energy savings in this step in our simulation study.

3) Cluster Join Announcement (CJA): Node, which is not a cluster head, joins a cluster with the nearest cluster head based on the received signal strength of the CHA message.

4) Frame Announcement (FA): Based on the number of client nodes in its cluster, cluster head creates a frame as illustrated in the Fig. 2. Frame is schedule of slots to each client node. Each client node transmits its data packet in its scheduled slot to the cluster head node.

In the data transfer phase, all the client nodes forward their data packets directly to its cluster head. Based on the required accuracy level and the type of data, cluster head aggregates the data packets. Cluster head forwards this aggregated packet to the sink node.

\section{Simulations}

In this section, we will evaluate our H-BAR protocol through extensive simulation study by comparing the $\mathrm{H}$ BAR and the LEACH protocols. We will make the following assumptions in our study.

- We assume that 100 sensor nodes are randomly placed over 100 X 100 square area sensor field.

- Each sensor node has a battery with 0.4 Jules of theoretical capacity and 0.2 Jules of nominal capacity and recovers 0.05 milli Jules of nominal capacity in each idle slot.

- Each data transfer phase consists of 500 slots.

- Size of the data packet is 250 bits and that of BSA control packet size is 25 bits.

- Cluster head aggregates 5 data packets into one packet before forwarding it to the sink node.

- Battery parameter, $g$, value is equal to $0.05 /$ milli Jule [15].

Graph shown in the Fig. 4 compares the lifetime of the WSN with our H-BAR and LEACH protocols [6], [7]. Based on the type of application and the coverage requirement, lifetime of the WSN can be defined as the number periods before a given percentage nodes dead. Notice that, a sensor node with zero nominal battery capacity is dead, even with nonzero theoretical battery capacity. In the graph shown in the
Fig. 4, unit on the $\mathrm{x}$-axis is the percentage of dead nodes and the unit on the $y$-axis is the number of periods.

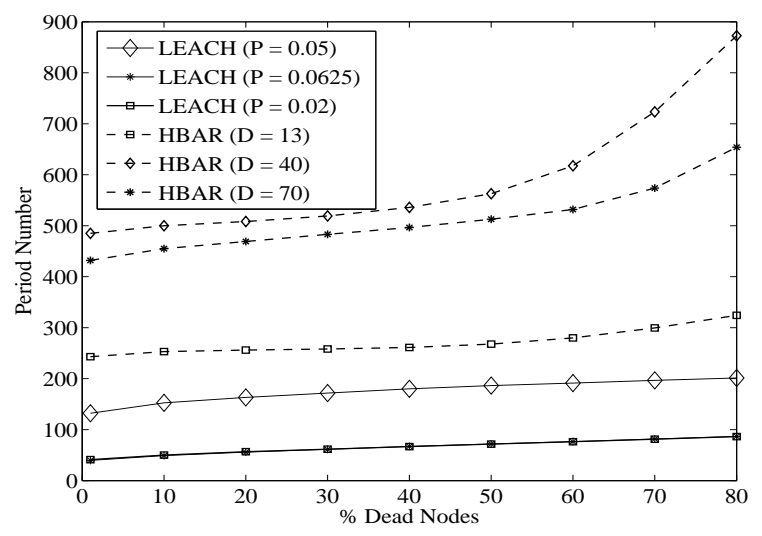

Fig. 4. Life-time of the WSN

In the LEACH protocol, CHA message, in the step 2, discharges much higher energy than our H-BAR protocol. But, we did not included these energy savings due to CHA message in our simulation study. Control overhead due to CJA message, in step 3, and FA message, in step 4, are the same in both LEACH and H-BAR protocols. Hence, we ignored the overhead due to these control messages in our simulation. In the H-BAR, BSA message, in the step 1, is a extra control message. We incorporated this extra control overhead due to BSA message in our simulation.

LEACH controls the average number of cluster heads per period by choosing a desired percentage of cluster heads $(P)$ value, which is an input parameter to the protocol. LEACH protocol, on average per period, chooses $P$ percentage of nodes as the cluster heads. As the $P$ value is increased from zero to a maximum value, the lifetime of the WSN reaches a maximum value before starts decreasing. From the simulations, LEACH with the battery recovery has a maximum lifetime value, when the $P$ value is equal to 0.05 . Similar result was noticed in [6], [7] without considering the battery recovery. In the graph shown in the Fig. 4, we showed the lifetime of the WSN with LEACH protocol for three $P$ values, i.e., when the $P$ value is equal to $0.02,0.05$ and 0.0625 . Lifetime of the LEACH protocol is maximum, when the $P$ is equal to 0.05 and the lifetime is lower, when the $P$ is equal to 0.02 and 0.0625 .

Similar to $\mathrm{LEACH}$, as the $D$ value increased from zero to maximum value, lifetime of the H-BAR reaches a maximum value before starts decreasing. In the graph shown in the Fig. 4, we showed the lifetime of the WSN with H-BAR protocol for three $D$ values, i.e., when the $D$ value is equal to 13,40 and 70. H-BAR has a maximum lifetime, when the $D$ value is equal to 40 and the lifetime of the WSN is lower, when $D$ is equal to 13 and 70. From the graph shown in the Fig. 4, H-BAR clearly improves the lifetime of the network up to 3 times over the probabilistic LEACH protocol.

Next, we will present the results to explain the reason for the better performance of the H-BAR protocol. As discussed 


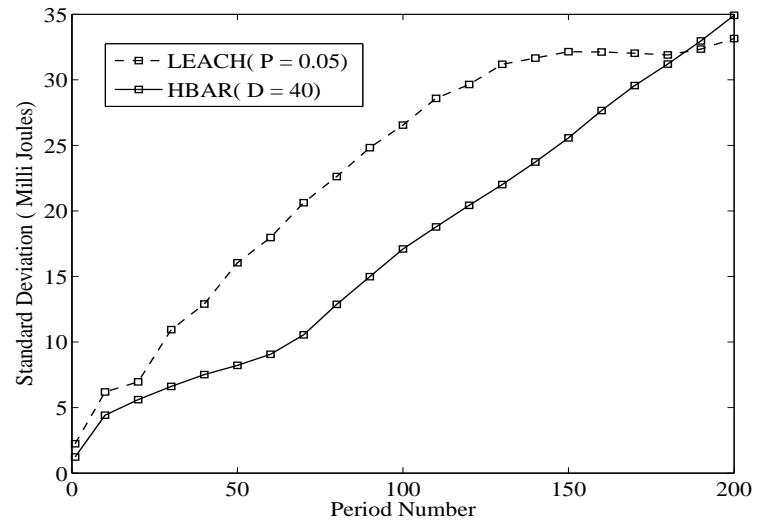

Fig. 5. Standard Deviation of $T$ in the WSN

before, the LEACH protocol does not discharge the battery uniformly from all the nodes. Next, graph shown in the Fig. 5 compares the standard deviation in the nominal battery capacities at different times during the simulation. In particular, we compared the H-BAR protocol, with $D$ value equal to 40 , and the LEACH protocol, with $P$ value equal to 0.05 , during the first 200 periods. LEACH protocol has a higher deviation than the H-BAR protocol, which decreases the amount battery capacity recovered in the $\mathrm{LEACH}$ protocol.

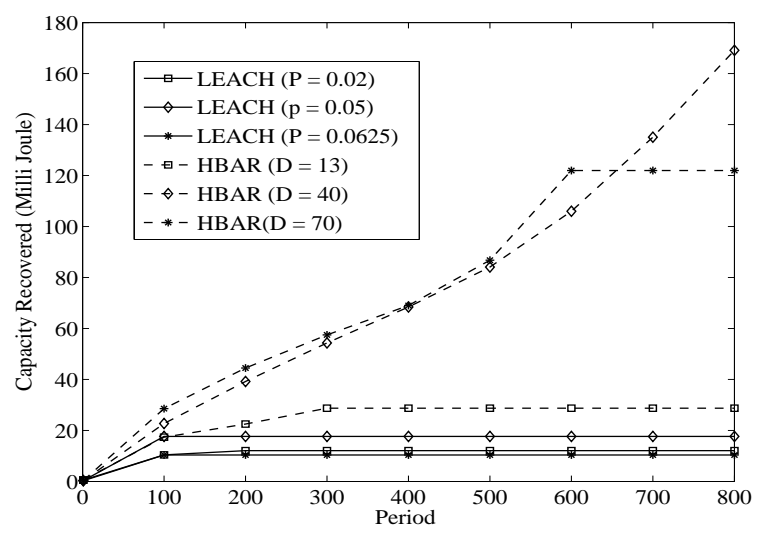

Fig. 6. Recovered Capacity in the WSN

Graph shown in the Fig. 6 compares the cumulative average nominal capacities recovered during 800 periods of simulation. We compared the LEACH protocol (with $P$ values $0.02,0.05$, and 0.0625 ), and the H-BAR protocol (with $D$ values 13,40 , and 70). From the graph shown in the Fig. 4, we know that the H-BAR, with $D$ value equal to 40 , has the highest lifetime. Reason for the highest lifetime is due to its highest battery capacity recovered, which can be observed from the graph shown in the Fig. 6. Similar observations can be made in other cases of the simulations.

\section{Summary AND CONCLUDING REMARKS}

The WSN have been envisioned to help in numerous monitoring applications. Energy efficient routing is paramount to extend the lifetime of the WSNs. In this paper, we proposed a Hierarchical-Battery Aware Routing (H-BAR) protocol. Our protocol uses the discharge properties of the battery to improve the battery capacities due to recovery capacity effect. Through extensive simulation study, we proved that our $\mathrm{H}-$ BAR protocol performs better than the probabilistic LEACH protocol.

In future, we want to develop an analytical model for the lifetime of the network. We also want to extend our protocol to heterogeneous WSNs [8].

\section{REFERENCES}

[1] I. F. Akyildiz, W. Su, Y. S. Subramaniam, and E. Cayirci, "Wireless sensor networks: a survey," Computer Networks, vol. 38, pp. 393-422, 2002.

[2] S. Gandham, R. Musunuri, , P. Rentala, , and U. Saxena, "A survey on self-organizing wireless sensor networks," in The Industrial Information Technology Handbook. CRC Press, 2004, pp. 78.1-78.16.

[3] E. J. Podlaha and H. Y. Cheh, "Modeling of cylindrical alkaline cells, vi. variable discharge conditions," vol. 141, 1994, pp. 28-35.

[4] R. F. Nelson, R. Rinehart, and S. Varley, "Ultrafast pulse discharge and recharge capabilities of thin-metal fi $\mathrm{lm}$ battery technology," in Proc. of IEEE IPPC Conference, vol. 1, Baltimore, 1997, pp. 636-641.

[5] C. F. Chiasserini and R. R. Rao, "Pulsed battery discharge in communication devices," in Proc. of ACM MOBICOM Conference, Seattle, 1999, pp. 88-95.

[6] W. Heinzelman, H. Balakrishnan, and A. Chandrakasan, "Energyeffi cient communication protocol for wireless microsensor networks," in Proc. of HICSS Conference, Maui, Hawaii, 2000, pp. 3005 - 3014.

[7] W. B. Heinzelman, A. P. Chandrakasan, and H. Balakrishnan, "An application-specifi c protocol architecture for wireless microsensor networks," vol. 1, no. 4, October 2002, pp. 660-670.

[8] G. Smaragdakis, I. Matta, and A. Bestavros, "SEP: A stable election protocol for clustered heterogenous wireless sensor networks," in Proc. of International Workshop on SANPA, Boston, 2004.

[9] C. Intanagonwiwat, R. Govindan, and D. Estrin, "Directed diffusion: A scalable and robust communication paradigm for sensor networks," in Proc. of the Int. Conf. on Mobile Computing and Net., Boston, 2000, pp. 56-67.

[10] S. Gandham, M. Dawande, R. Prakash, and S. Venkatesan, "Energy effi cient schemes for wireless sensor networks with multiple mobile base stations," in Proc. of IEEE GLOBECOM Conference, vol. 1, San Francisco, 2003, pp. 377 - 381.

[11] D. Linden and T. B. Reddy, in Handbook of Batteries. McGraw-Hill, 2002.

[12] C. F. Chiasserini and R. R. Rao, "A traffi c control scheme to optimize the battery pulsed discharge," in Proc. of IEEE MILCOM Conference, vol. 2, Atlantic City, 1999, pp. 1419 - 1423.

[13] J. Elson and D. Estrin, "Time synchronization for wireless sensor networks," in Proc. of International Parallel and Distributed Processing Symposium, San Francisco, CA, 2001, pp. 1965 - 1970.

[14] M. L. Sichitiu and C. Veerarittiphan, "Simple, accurate time synchronization for wireless sensor networks," in Proc. of the IEEE WCNC Conference, New Orleans, LA, 2003.

[15] J. Subramanian, B. S. Manoj, and S. R. Murthy, "On using battery state for medium access control in ad hoc wireless networks," in Proc. of ACM MOBICOM Conference, Philadelphia, 2004, pp. 360 - 373.

[16] C. F. Chiasserini and R. R. Rao, "Energy effi cient battery management," in Proc. of IEEE Infocom Conference, vol. 2, Tel Aviv, Israel, 2000, pp. 396-403.

[17] - , "Improving battery performance by using traffi c shaping techniques," vol. 19, no. 7, July 2001, pp. 1385-1394.

[18] S. Bandyopadhyay and E. J. Coyle, "An energy effi cient hierarchical clustering algorithm for wireless sensor networks," in Proc. of IEEE INFOCOM Conference, vol. 3, San Francisco, 2003, pp. 1713 - 1723. 